\section{Primeira etapa da adaptação transcultural do instrumento The Vulnerable Elders Survey (VES-13) para o Português}

\author{
First stage of the cross-cultural adaptation of the \\ instrument The Vulnerable Elders Survey (VES-13) \\ to Portuguese
Primera etapa de la adaptación transcultural del instrumento The Vulnerable Elders Survey (VES-13) al portugués

Laércio Lima Luz 1

Lívia Maria Santiago 2

João Francisco Santos da Silva ${ }^{3}$

Inês Echenique Mattos 1

\footnotetext{
${ }^{1}$ Escola Nacional de Saúde Pública Sergio Arouca, Fundação Oswaldo Cruz, Rio de Janeiro, Brasil.

2 Faculdade de Medicina, Universidade Federal do Rio de Janeiro, Rio de Janeiro, Brasil.

3 Secretaria de Estado de Saúde do Mato Grosso do Sul, Campo Grande, Brasil.

Correspondência L. L. Luz

Escola Nacional de Saúde Pública Sergio Arouca, Fundação Oswaldo Cruz. Rua Leopoldo Bulhões 1480 , Rio de Janeiro, RJ 21041-210, Brasil.

laercioll@hotmail.com
}

\begin{abstract}
The aim of this study was to describe the initial stages of the cross-cultural adaptation of the instrument The Vulnerable Elders Survey (VES-13) for use in the context of cancer care in Brazil. Two translations into Portuguese and two back-translations into English were carried out independently, and a formal assessment of the general and referential meanings was performed in order to obtain a synthesis version. Understanding of the synthesis version was evaluated in a pretest applied to 33 patients in an oncologic hospital of the Brazilian Unified National Health System (SUS). The version was easily applied in the intended context and was well-accepted by elders. The Portuguese version of the VES-13 proved to be well understood and adequate for testing its psychometric qualities. The latter step is currently in the final phase.
\end{abstract}

Geriatric Assessment; Neoplasms; Aged; Vulnerable Population

\section{Resumo}

O objetivo do estudo foi descrever as etapas iniciais de adaptação transcultural do instrumento The Vulnerable Elders Survey (VES-13) para utilização no contexto da assistência oncológica no Brasil. Foram realizadas, de modo independente, traduções para o português e retraduções para o inglês. Procedeu-se a avaliação formal dos significados geral e referencial chegando-se a uma versão síntese. A compreensão da versão síntese foi avaliada em pré-teste aplicado a 33 pacientes em um hospital de oncologia habilitado pelo Sistema Único de Saúde. Observou-se facilidade de aplicação no contexto pretendido e boa aceitação entre os idosos. Considerou-se que a versão em português do VES-13 foi bem compreendida e se mostrou adequada para ser testada em suas qualidades psicométricas. Essa etapa encontra-se atualmente em fase de conclusão.

Avaliação Geriátrica; Neoplasias; Idoso; Populações Vulneráveis 


\section{Introdução}

O The Vulnerable Elders Survey (VES-13) foi desenvolvido nos Estados Unidos, utilizando dados da população de 65 anos ou mais usuária do Medicare, e tinha o objetivo de rastrear idosos com risco de deterioração da saúde 1. É um instrumento simplificado, com tempo médio de aplicação de cinco minutos e constituído por 13 itens, que contemplam idade, saúde autorreferida, capacidade funcional e condição física 1. Apresentou boa confiabilidade e validade em estudo com idosos com câncer de próstata 2 , e tem sido utilizado como instrumento de rastreio em pacientes oncológicos 3,4 .

O objetivo deste trabalho foi descrever as primeiras etapas da adaptação transcultural do VES-13 para a população brasileira e apresentar uma versão síntese para posterior avaliação de suas propriedades psicométricas.

\section{Metodologia}

A adaptação transcultural do instrumento VES13 baseou-se na abordagem universalista, sendo aqui descritas as três primeiras etapas 5,6 (Figura 1).

$\mathrm{O}$ constructo de interesse foi explorado em revisão de literatura que forneceu elementos para discussão da equivalência conceitual. Um grupo multidisciplinar com especialistas nas áreas de epidemiologia, câncer e envelhecimento analisou a relevância da dimensão avaliada pelo instrumento original e a pertinência dos itens no contexto cultural da população idosa brasileira.

Dois médicos brasileiros com fluência no idioma inglês e conhecedores dos objetivos do instrumento realizaram, de modo independente, duas traduções do instrumento original para o português (T1 e T2). Essas versões foram retraduzidas para o inglês (R1 e R2), também de modo independente, por dois profissionais proficientes nos dois idiomas e que não tiveram acesso ao instrumento original.

Uma médica geriatra, com formação em saúde coletiva e proficiente em inglês, realizou a avaliação formal das retraduções em relação ao instrumento original. Quanto ao significado referencial de cada item, a avaliadora recebeu um formulário e lhe foi solicitado que atribuísse pontuação entre 0 e $100 \%$ de acordo com a correspondência literal. Outro formulário foi utilizado para avaliar o significado geral dos itens e qualificá-los nas categorias: inalterado; pouco alterado; muito alterado e; completamente alterado.

O grupo multidisciplinar discutiu a avaliação dos significados referencial e geral e sugeriu mo- dificações em alguns itens para melhorar a compreensão e objetividade.

A compreensibilidade da versão síntese foi avaliada por meio de pré-teste em um hospital de oncologia do SUS em Campo Grande (Mato Grosso do Sul). Foram entrevistados 33 pacientes com câncer, sendo 54,5\% do sexo masculino, com idade média de $69,61( \pm 1,07)$ anos e predomínio de baixa escolaridade ( $\leq 4$ anos). Solicitouse que os indivíduos parafraseassem cada item da versão síntese. Estabeleceu-se previamente que itens incompreendidos por $15 \%$ ou mais dos entrevistados seriam objeto de discussão para reformulação.

\section{Resultados}

Com base na revisão de literatura e nas discussões da equipe, concluiu-se que o conceito de idoso vulnerável é relevante na cultura brasileira, tendo a mesma concepção nos diferentes países que realizam estudos na área de oncologia geriátrica 3,4 .

Na Tabela 1, observa-se boa equivalência entre os itens da versão original e das retraduções. A maior parte teve seu significado referencial avaliado entre $80 \%$ e $95 \%$ em relação ao original. Os itens 3 e 4 apresentaram maior discrepância, respectivamente em R1 e R2. O significado geral foi considerado inalterado ou pouco alterado para todos os itens, com exceção do 4 (R2). Não se observaram discrepâncias quanto aos significados geral e referencial das versões. Ainda que as duas traduções tenham sido consideradas adequadas, a equivalência semântica de R1 foi melhor que a de R2 e houve tendência para seleção dos itens de T1 na elaboração da versão síntese.

Quanto às opções de respostas, as da T1 foram consideradas mais próximas do original e refletiam melhor seu conteúdo, sendo selecionadas para o item 2 e para os itens de 3 a 8.

Na versão original, o enunciado principal referente aos itens de 3 a 8 aparece apenas uma vez antes do item 3. Julgou-se apropriado repeti-lo antes de todos os itens para facilitar a aplicação no contexto pretendido. Procedeu-se da mesma forma com os itens de 9 a 13 .

Nesses itens, dependendo da resposta à pergunta inicial, o entrevistado é indagado se tem ajuda para realizar a atividade ou se não consegue realizá-la devido à sua saúde. Todas as subperguntas dos itens de 9 a 12 foram mantidas como se encontravam em $\mathrm{T} 1$.

Na Tabela 2, encontram-se os itens selecionados, suas respectivas origens e a versão síntese submetida ao pré-teste. 


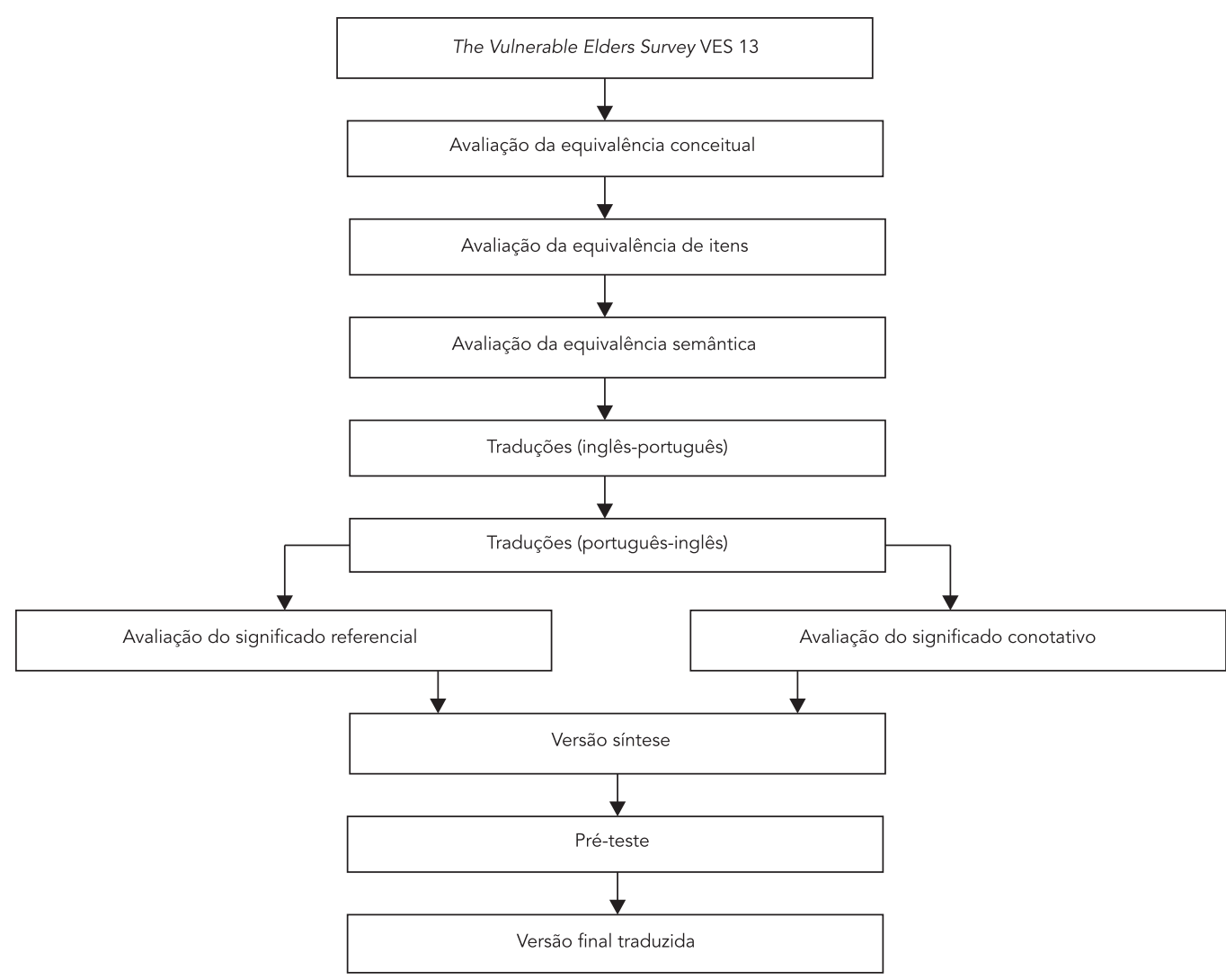

No instrumento original o item 1 é composto apenas pela palavra "age". Optou-se por perguntar ao indivíduo "qual é a sua idade?”, para tornar a pergunta mais objetiva.

Nos itens de 3 a 8 suprimiu-se do enunciado da versão selecionada as expressões "em geral" e "realizar as seguintes atividades", que tornavam a assertiva longa, sem acrescentar informações relevantes.

A unidade de medida de peso do item 4 foi convertida para uma unidade utilizada em nosso meio, o quilograma. Para facilitar a compreensão, aproximou-se o valor de 4,5 para $5 \mathrm{~kg}$, visto que isto pouco alteraria o significado.

Foi consenso entre os especialistas que a retirada da palavra "altura" no item 5 não modificaria o sentido da pergunta e a tornaria mais objetiva.

No item 6, decidiu-se inverter a posição dos termos "objetos" e "pequenos" por ser esta a construção usual no português.
A unidade de medida utilizada no item 7, a milha, não é padrão em nosso meio, sendo necessária a sua conversão para metros.

Nos itens de 9 a 13, a versão escolhida continha a expressão "devido a”. Na discussão, preferiu-se substituí-la pela expressão "por causa", cujo significado referencial se aproxima mais do original. Optou-se por retirar a palavra "alguma", por nada acrescentar ao constructo.

No item 9, o termo "pessoal" foi retirado da expressão entre parênteses por ser desnecessária a sua repetição. No item 12, substituiu-se o termo "realizar" por "fazer", que daria mais clareza à assertiva.

Quanto ao item 13, concluiu-se que em nosso contexto o termo "banho" tem significado mais amplo, abrangendo tanto o uso do chuveiro quanto o da banheira. Optou-se por utilizar somente a palavra "banho" no questionamento principal e no subitem. 
Tabela 1

Comparação entre o original em inglês e as retraduções do instrumento The Vulnerable Elders Survey (VES-13).

\begin{tabular}{cc}
\hline Item & Original \\
\hline 1 & Age \\
2 & $\begin{array}{c}\text { In general, compared to other } \\
\text { people your age, would you say } \\
\text { that your health is: }\end{array}$ \\
& How much difficulty, on average, \\
& do you have with the following \\
& physical activities: stooping, \\
& crouching or kneeling?
\end{tabular}

4 [How much difficulty, on average, do you have with the following physical activities:] lifting, or carrying objects as heavy as 10 pounds?

5 [How much difficulty, on average, do you have with the following physical activities:] reaching or extending arms above shoulder level?

6 [How much difficulty, on average, do you have with the following physical activities:] writing, or handling and grasping small objects?

7 [How much difficulty, on average, do you have with the following physical activities:] walking a quarter of a mile?

8 [How much difficulty, on average, do you have with the following physical activities:] heavy housework such as scrubbing floors or washing windows?

9 Because of your health or a physical condition, do you have any difficulty: shopping for personal items (like toilet items or medicines)?

10 [Because of your health or a physical condition, do you have any difficulty:] managing money (like keeping track of expenses or paying bills)?

11 [Because of your health or a physical condition, do you have any difficulty:] walking across the room?

T1/R11
Age
In general in comparison with other
people your age, would you say
that your health is:
Which degree of difficulty do you
usually have with the following
physical activities? To bend over,
kneel or crouch?

Which degree of difficulty do you usually have with the following physical activities? To lift or carry things that weigh around 10

$$
\text { pounds? }
$$

Which degree of difficulty do you usually have with the following physical activities? Reaching or extending arms above shoulder level?

Which degree of difficulty do you usually have with the following physical activities? To write or to hand over or catch small objects?

Which degree of difficulty do you usually have with the following physical activities? To walk around a quarter mile?

Which degree of difficulty do you usually have with the following physical activities? To perform heavy work activities such as scrubbing the floors or washing windows?

Because of your health or physical condition do you have any difficulty in: Shopping for personal items (hygiene products, medication)?

Because of your health or physical condition do you have any difficulty in: Handling your finances (managing your expenses or paying bills)?

Because of your health or physical condition do you have any difficulty in: Walking across the living room?

\begin{tabular}{|c|c|c|c|c|}
\hline $\mathrm{R}^{2}$ & $\mathrm{G}^{3}$ & T2/R24 & $\mathrm{R}^{2}$ & $\mathrm{G}^{3}$ \\
\hline 100 & IN & Age & 100 & IN \\
\hline 95 & IN & $\begin{array}{c}\text { Overall, compared to other people } \\
\text { of your age, would you say your } \\
\text { health is: }\end{array}$ & 90 & PA \\
\hline 60 & PA & $\begin{array}{c}\text { On average, how much trouble } \\
\text { do you have with the following } \\
\text { physical activities: bending, } \\
\text { squatting or kneeling? }\end{array}$ & 70 & PA \\
\hline 70 & PA & $\begin{array}{l}\text { On average, how much trouble } \\
\text { do you have with the following } \\
\text { physical activities: Lifting or } \\
\text { carrying objects as heavy as four } \\
\text { and a half pounds? }\end{array}$ & 60 & MA \\
\hline 80 & IN & $\begin{array}{l}\text { On average, how much trouble } \\
\text { do you have with the following } \\
\text { physical activities: Reaching or } \\
\text { extending your arms above your } \\
\text { shoulders? }\end{array}$ & 90 & IN \\
\hline 80 & PA & $\begin{array}{c}\text { On average, how much trouble } \\
\text { do you have with the following } \\
\text { physical activities: Writing or } \\
\text { handing over and grasping small } \\
\text { objects? }\end{array}$ & 95 & IN \\
\hline 80 & PA & $\begin{array}{c}\text { On average, how much trouble } \\
\text { do you have with the following } \\
\text { physical activities: Walking about } \\
400 \text { meters? }\end{array}$ & 80 & PA \\
\hline 80 & PA & $\begin{array}{c}\text { On average, how much trouble } \\
\text { do you have with the following } \\
\text { physical activities: Doing some } \\
\text { heavy house chores such as } \\
\text { scrubbing floors or washing the } \\
\text { windows? }\end{array}$ & 80 & PA \\
\hline 95 & PA & $\begin{array}{l}\text { Due to your health or physical } \\
\text { condition, do you have any } \\
\text { difficulty: Purchasing items for } \\
\text { personal use (such as toiletries or } \\
\text { medicines)? }\end{array}$ & 80 & PA \\
\hline 80 & PA & $\begin{array}{l}\text { Due to your health or physical } \\
\text { condition, do you have any } \\
\text { difficulty: Dealing with your money } \\
\text { (such as keeping track of your } \\
\text { expenses or paying bills)? }\end{array}$ & 70 & PA \\
\hline 95 & IN & $\begin{array}{l}\text { Due to your health or physical } \\
\text { condition, do you have any } \\
\text { difficulty: Walking around the } \\
\text { room? }\end{array}$ & 80 & PA \\
\hline
\end{tabular}

(continua) 
Tabela 1 (continuação)

\begin{tabular}{|c|c|c|c|c|c|c|c|}
\hline Item & Original & T1/R11 & $\mathrm{R}^{2}$ & $\mathrm{G}^{3}$ & T2/R24 & $\mathrm{R}^{2}$ & $\mathrm{G}^{3}$ \\
\hline 12 & $\begin{array}{l}\text { [Because of your health or a } \\
\text { physical condition, do you have any } \\
\text { difficulty:] doing light housework } \\
\text { (like washing dishes, straightening } \\
\text { up, or light cleaning)? }\end{array}$ & $\begin{array}{c}\text { Because of your health or physical } \\
\text { condition do you have any difficulty } \\
\text { in: Performing simple chores } \\
\text { (like washing the dishes or light } \\
\text { cleaning)? }\end{array}$ & 80 & PA & $\begin{array}{l}\text { Due to your health or physical } \\
\text { condition, do you have any } \\
\text { difficulty: Doing light house chores } \\
\text { (like washing dishes, tidying up the } \\
\text { house or doing light cleaning)? }\end{array}$ & 70 & PA \\
\hline 13 & $\begin{array}{l}\text { [Because of your health or a } \\
\text { physical condition, do you have any } \\
\text { difficulty:] bathing or showering? }\end{array}$ & $\begin{array}{c}\text { Because of your health or physical } \\
\text { condition do you have any difficulty } \\
\text { in: Taking a bath/shower? }\end{array}$ & 90 & IN & $\begin{array}{c}\text { Due to your health or physical } \\
\text { condition, do you have any } \\
\text { difficulty: Taking a bath or shower? }\end{array}$ & 85 & IN \\
\hline
\end{tabular}

G3: significado geral; IN: inalterado; MA: muito alterado; PA: pouco alterado; R2: significado referencial; T1/R11: retradução para o inglês da versão 1 em português; T2/ R24: retradução para o inglês da versão 2 em português.

No pré-teste observou-se que o instrumento foi de fácil aplicação no contexto pretendido e que obteve boa aceitação pelos idosos. Quanto à compreensão dos itens, subitens e opções de resposta, somente dois idosos entrevistados tiveram alguma dificuldade no entendimento, um no item 6 e outro no item 7.

$\mathrm{Na}$ avaliação final do pré-teste, o grupo de especialistas considerou apropriada a realização de duas modificações pontuais. No item 2 substituiu-se “...você diria que sua saúde é” por “... como é a sua saúde?”, pois foi desta forma que a maioria dos entrevistados parafraseou a questão. No item 8, optou-se pela troca do verbo "lavar" por "limpar", por ser o mais adequado para expressar a atividade em nosso meio.

Após esses ajustes semânticos considerou-se a versão síntese do instrumento pronta para ser submetida à avaliação de suas propriedades psicométricas (Figura 2).

\section{Discussão}

A realização de uma avaliação geriátrica multidimensional é recomendada em oncologia para a definição do protocolo de tratamento de pacientes idosos ${ }^{2}$. Por ser extensa e demorada, o rastreamento com um instrumento de fácil aplicação tem sido proposto para identificar aqueles que necessitariam de avaliação mais completa 2,3,4. Esse é o objetivo do VES-13: rastrear os idosos vulneráveis, ou seja, aqueles com maior risco de declínio funcional e morte, para que sejam submetidos a uma avaliação mais ampla 1 .

O conceito de vulnerabilidade tem correspondência na cultura brasileira e é empregado em estudos com idosos para caracterizar indivíduos em maior risco nos diferentes contextos $3,4,7$.

Os itens que constituem o VES-13 avaliam atividades comuns do cotidiano e a revisão da literatura mostrou que têm interpretação similar nas duas culturas 3,4,8. A avaliação dos significados geral e referencial mostrou poucas discrepâncias entre o instrumento original e as retraduções.

A discussão do grupo na etapa de elaboração da versão síntese propiciou a reformulação de alguns itens, tornando-os mais concisos e coloquiais, facilitando a compreensibilidade e aceitação do instrumento pela população-alvo.

Ao solicitarmos que os indivíduos parafraseassem os itens do instrumento, ficou evidenciada a compreensão adequada do significado dos mesmos e a semelhança entre a versão proposta e a original em inglês. Mesmo assim, ao avaliar as paráfrases dos entrevistados, o grupo considerou oportuna a realização de pequenos ajustes semânticos para tornar a linguagem do instrumento ainda mais próxima daquela da população-alvo.

No estudo que descreve o desenvolvimento do instrumento original, os autores não indicaram uma forma específica de aplicação ${ }^{1}$. Na literatura consultada diferentes formas foram utilizadas. Optou-se pela entrevista face a face, considerando a baixa escolaridade da população idosa a ser avaliada.

A versão brasileira do VES-13 foi bem compreendida e se mostrou adequada para a realização de testes de suas qualidades psicométricas, de forma a avaliar sua utilidade no rastreamento de idosos vulneráveis na assistência oncológica. 
Tabela 2

Origem dos itens selecionados e versão síntese (pré-teste).

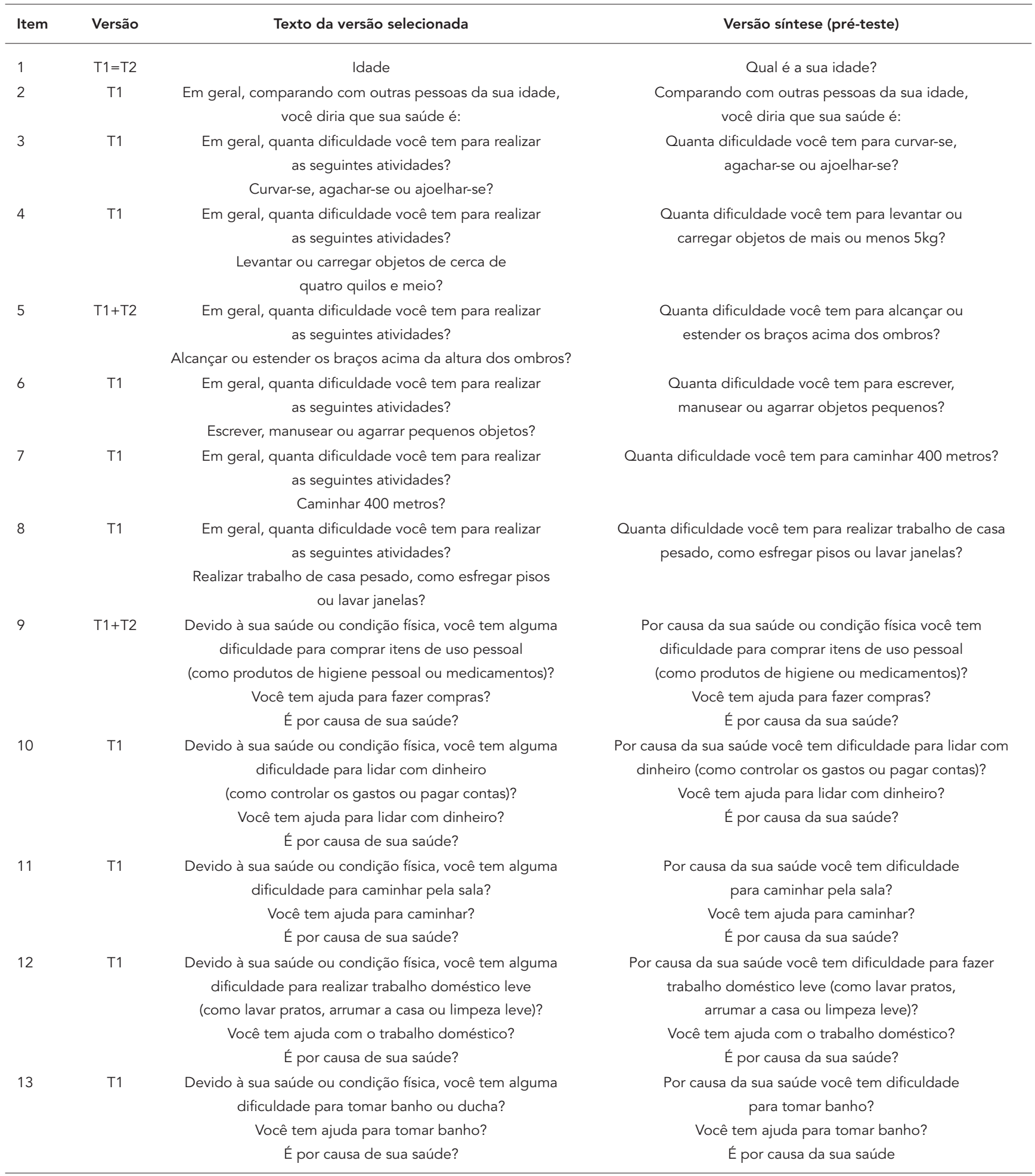

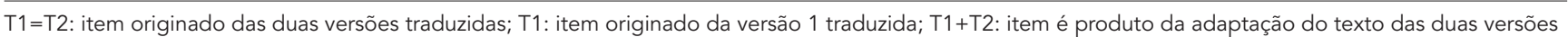
traduzidas. 
Versão pós pré-teste do instrumento The Vulnerable Elders Survey (VES-13).

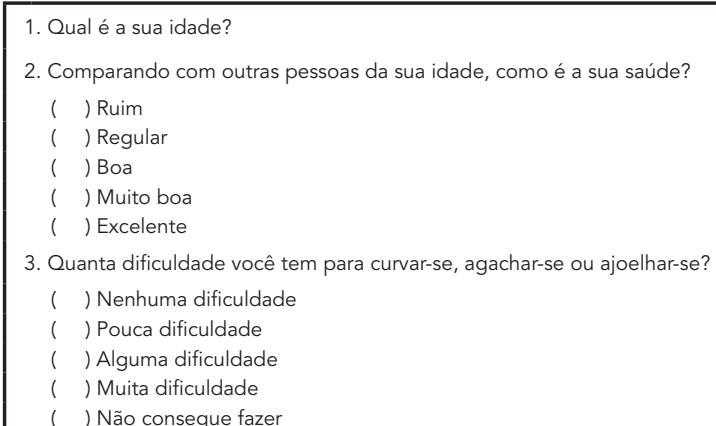

4. Quanta dificuldade você tem para levantar ou carregar objetos de mais ou menos $5 \mathrm{~kg}$ ?
( ) Nenhuma dificuldade
( ) Pouca dificuldade
( ) Alguma dificuldade
( ) Muita dificuldade
( ) Não consegue fazer

5. Quanta dificuldade você tem para alcançar ou estender os braços acima dos ombros?
( ) Nenhuma dificuldade
( ) Pouca dificuldade
( ) Alguma dificuldade
( ) Muita dificuldade
( ) Não consegue fazer

6. Quanta dificuldade você tem para escrever, manusear ou agarrar objetos pequenos?
( ) Nenhuma dificuldade
( ) Pouca dificuldade
( ) Alguma dificuldade
( ) Muita dificuldade
( ) Não consegue fazer

7. Quanta dificuldade você tem para caminhar 400 metros?
( ) Nenhuma dificuldade
( ) Pouca dificuldade
( ) Alguma dificuldade
( ) Muita dificuldade
( ) Não consegue fazer

8. Quanta dificuldade você tem para realizar trabalho de casa pesado, como esfregar pisos ou limpar janelas?

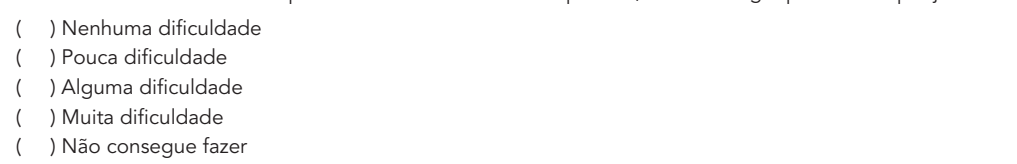

9. Por causa da sua saúde ou condição física você tem dificuldade para comprar itens de uso pessoal (como produtos de higiene ou medicamentos)?

( ) SIM. Você tem ajuda para fazer compras? ( ) SIM ( ) NÃO

( ) NÃO

( ) NÃO FAZ. É por causa da sua saúde? ( ) SIM ( ) NÃO

10. Por causa da sua saúde você tem dificuldade para lidar com dinheiro (como controlar os gastos ou pagar contas)?

( ) SIM. Você tem ajuda para lidar com dinheiro? ( ) SIM （） NÃO

( ) NÃO

( ) NÃO FAZ. É por causa da sua saúde? ( ) SIM ( ) NÃO

11. Por causa da sua saúde você tem dificuldade para caminhar pela sala?

( ) SIM. Você tem ajuda para caminhar? ( ) SIM ( ) NÃO

( ) NÃO

( ) NÃO FAZ. É por causa da sua saúde? ( ) SIM （） NÃO

12. Por causa da sua saúde você tem dificuldade para fazer trabalho doméstico leve (como lavar pratos, arrumar a casa ou limpeza leve)?

( ) SIM. Você tem ajuda com o trabalho doméstico? ( ) SIM ( ) NÃO

( ) NÃO

( ) NÃO FAZ. É por causa da sua saúde? ( ) SIM （） NÃO

13. Por causa da sua saúde você tem dificuldade para tomar banho?

( ) SIM. Você tem ajuda para tomar banho? ( ) SIM ( ) NÃO

( ) NÃO

( ) NÃO FAZ. É por causa da sua saúde? ( ) SIM ( ) NÃO 


\section{Resumen}

El objetivo del estudio fue describir las etapas iniciales de la adaptación transcultural del instrumento The Vulnerable Elders Survey (VES-13) para su uso en el contexto de la atención del cáncer en Brasil. Se realizaron, de forma independiente, las traducciones al portugués con el apoyo de traducciones al inglés. Se procedió a la evaluación formal de los significados generales y referenciales, llegándose a una versión resumida. La comprensión de la versión sintética se evaluó en el pre-test administrado en 33 pacientes de un hospital oncológico, habilitado por el Sistema Único de Salud. Se facilitó la aplicación en el contexto previsto y hubo una buena aceptación entre los ancianos. Se considera que la versión portuguesa de la VES-13 es inteligible y adecuada para ser evaluada en su apartado psicométrico. Esta fase está a punto de finalizar.

Evaluación Geriátrica; Neoplasias; Anciano; Poblaciones Vulnerables

\section{Colaboradores}

L. L. Luz garantiu a integridade do estudo, o conceito da investigação, o delineamento, a pesquisa bibliográfica, a análise crítica e estatística dos dados, e a elaboração do manuscrito. L. M. Santiago foi responsável pelo conceito de estudo, definição de conteúdo intelectual, análise dos dados e revisão do manuscrito. J. F. S. Silva foi responsável pelo conceito de estudo, definição de conteúdo intelectual, análise dos dados e revisão do manuscrito. I. E. Mattos foi responsável pelo conceito da investigação, delineamento, definição de conteúdo intelectual, análise dos dados, edição do manuscrito/ revisão.

\section{Agradecimentos}

Este trabalho contou com o apoio financeiro do Programa de Desenvolvimento Tecnológico em Saúde Publica (PDTSP) da Fundação Oswaldo Cruz/Conselho Nacional de Desenvolvimento Cientifico e Tecnológico (CNPq).

\section{Referências}

1. Saliba D, Elliott M, Rubenstein LZ, Solomon DH, Young RT, Kamberg CJ, et al. The Vulnerable Elders Survey: a tool for identifying vulnerable older people in the community. J Am Geriatr Soc 2001; 49:1691-9.

2. Mohile SG, Bylow K, Dale W, Dignam J, Martin K, Petrylak DP, et al. A pilot study of the Vulnerable Elders Survey-13 compared with the Comprehensive Geriatric Assessment of identifying disability in older patients with prostate cancer who receive androgen ablation. Cancer 2007; 109:802-10.

3. Kellen E, Bulens P, Deckx L, Schouten H, Van Dijk $\mathrm{M}$, Verdonck I, et al. Identifying an accurate prescreening tool in geriatric oncology. Crit Rev Oncol Hematol 2010; 75:243-8.

4. Luciani A, Ascione G, Bertuzzi C, Marussi D, Codecà C, Di Maria G, et al. Detecting disabilities in older patients with cancer: Comparison between Comprehensive Geriatric Assessment and Vulnerable Elders Survey-13. J Clin Oncol 2010; 28: 2046-50.
5. Herdman M, Fox-Rushby J, Badia X. A model of equivalence in the cultural adaptation of HRQoL instruments: the universalist approach. Qual Life Res 1998; 7:323-35.

6. Reichenheim ME, Moraes CL. Operacionalização de adaptação transcultural de instrumentos de aferição usados em epidemiologia. Rev Saúde Pública 2007; 41:665-73.

7. Paz AA, Santos BR, Eidt OR. Vulnerabilidade e envelhecimento no contexto da saúde. Acta Paul Enferm 2006; 19:338-42.

8. Lebrão ML, Laurenti R. Saúde, bem-estar e envelhecimento: o estudo SABE no Município de São Paulo. Rev Bras Epidemiol 2005; 8:127-41.

Recebido em 27/Mai/2012

Versão final reapresentada em 13/Set/2012 Aprovado em 13/Nov/2012 